\title{
A Literature Review of Wave-Induced Heave Motion of Floating Structures
}

\author{
School of Mechanical and Chemical Engineering, University of Western Australia whspxja@aliyun.com
}

\section{Introduction}

As the development and exploration of oil and gas industry expanding to deep sea area, more and more production systems are constructed. Floating platforms, the hardcore of the production system, is equipped with facilities to extract and process oil or natural gas and to house the workforce as well. Thus, the study regarding to the motion of those floating platform could be crucial for design consideration and energy conservation [1]. The common platforms include: spar platform, truss spar, tension-leg platforms etc..

Spar platforms, their good performance in deep sea condition make them very popular in the Mexico Gulf (GoM). Rho et al ${ }^{[2]}$. paid a lot attend to spar platforms, the team has studied the coupled heave-pitch motion of the spar platform and found that the it is the nonlinear mechanism lies in the heave-pitch mode effect the energy transfer phenomenon. Damping is also a problem for Spar platform that concerns engineers, in 2004, the viscous damping coefficients of spar platforms with changing geometries was calculated by Tao et. al $^{[3]}$ the research team found that the heave-resonant response can be significantly decreased by employing alternative hull shapes to obtain higher damping and change the natural heave frequency. Hong et al. ${ }^{\text {[4] }}$ studied the Mathieuin stability of the spar platform as well as the pitch that is associated with heave motion, they have proved that a coupling effect exists between heave and pitch for the spar platform. Neves et al. [5] employed a coupled non-linear mathematical model to simulate the coupled heave, roll and pitch motions and investigated the dynamic stability of a vertical cylinder in regular waves theoretically and experimentally. Zhao et al. [6] studied the internal resonance of the spar platform's heavepitch coupling motions in regular waves, and the results indicated that the energy of the heave mode was saturated when the wave height arrived at a certain value, as wave height increased further, the extra energy of the heave mode was transferred to the pitch mode. Shen et al. ${ }^{[7]}$ studied the heavepitch coupling motion response of the spar platform by considering 1st-order random wave loads. Investigations on the motion response of the spar platform mainly focus on the these two parts: 1st is the platform's heave and pitch motions existed in the vertical plane, 2nd is the vortex-induced motion of the platform existed in the horizontal plane ${ }^{[8]}$.

The truss spar is an updated product generated from spar platform that significantly reduces the wave loads on the body. The surge, heave and pitch motion response of truss spar are tested in a wave basin and then compared with the measured data with the results calculated by the MATLAB program by Kurian et al. ${ }^{[9]}$.

The review articles are in a very high level of study, but the aim of this review is focus on the basic offshore engineering concept regarding to the heave

\begin{abstract}
This paper provides comprehensive review on heave motion of rigid floating structure due to wave impacts. To specify and explain the structure response, this review firstly provides a brief introduction on ocean sea wave theory, floating structure motion interpretation. Then the floating body motion in regular waves was demonstrated using a superposition method of the oscillated motion in still water and the restrained motion in waves. Meanwhile, added mass and damping coefficient, these two frequency-dependent terms are brought into discussion to generate the motion response with given wave amplitude, which is known for response amplitude operator( RAO). Based on the study in regular waves, RAO of floating structure in irregular waves is introduced while no longer in time domain but in frequency domain. The whole review covers the literatures from the early 1980s up to nowadays, based on the review, it is recommended that more experimental work regarding to frequency characteristic and relative response of larger floating body should be carried out to improve the accuracy of this method.
\end{abstract}

Key words: Floating body; heave motion analysis; JONSWAP spectrum; Response Amplitude Operator (RAO); literature review

Published online: 31st January, 2018 
motion analysis. In 2009, Li and Ou [10] used numerical iteration and linearized viscous damping methods to obtain the heave Response Amplitude Operators (RAOs) of a truss spar with a variable number of heave plates. The team also obtained the heave response spectra in the frequency domain and found that the platform's heave was sensitive to the wave period. The method used in this report are same as Li's article. Hopefully, throughout a simple cylinder model, the report could demonstrate this numerical approach for motion response analysis.

Consider an irregular sea state, the irregular waves could be defined with an energy associated term over the wave frequencies, this term is called wave spectrum or energy density. It is a known quantity once the we have wave record. These waves are input for a linear body-wave system. The frequency response characteristics is like the transfer function for the system, which are obtained from, for instance via simulations or model experiments. The output of the system is the motion of the floating structure. Because the input is irregular, thus the motion response shall follow an irregular behavior. In many cases the ship motions mainly have a linear behavior. This means that, at each frequency, the different ratios between the motion amplitudes and the wave amplitudes and also the phase angles between the motions and the waves are constant. Normally, a very practical and realistic mathematical model of the motions in a seaway can be obtained by making use of a superposition of these components at a range of frequencies which is similarly to the way to generate the wave spectrum. The motion analysis is so-called frequency domain approach will be considered here. It is a common sense that, as a consequence of linear theory, the resulting motions in irregular waves can be obtained by sum up the results obtained from regular waves with different frequencies, amplitudes and propagating directions. With known wave spectrum and the calculated transfer function of the motion responses of the ship, the response motion spectrum and associated statistics of these responses can be found. In this report, this numerical method was thoroughly proposed to solve the heave of given objects with given wave data.

\section{Aim}

The thesis provides a literature review on heave motion analysis of floating structure as well as associated wave theories, aims to provide a comprehensible example to solve the problem numerically. This is achieved by summarizing the outcomes made by predecessor and extract the relevant ideas. Basically, the motion response is solved by following steps:

- Obtain the wave spectrum of given wave data

- Determine the Coefficients associated with RAO

- Compute Response Amplitude Operator(RAO) of floating body

- To specify and explain the structure response

\section{Ocean surface waves}

- It is necessary to have basic concept of waves before analysing floating body response because ocean surface waves are the causes for the loads on the floating structures. Waves from their generation could be classified into following types:

- Waves are most commonly generated by wind. Wind driven waves, which are created by the friction between wind and water surface.

- Waves generated by large meteorite strike, such as earthquakes or underwater landslide, are called tsunami.

- Waves generated by gravitational pull from sun and moon are called tides.

- Waves could also be generated by a ship or floating structure moving at the surface.

Among these types, the winddriven waves are major concern for engineers in most research. J.M.J. Journée and W.W. Massie stated that in order to analyze the complicated sea state, it is necessary to know the properties of the single regular component, such as time and locationdependent pressure in the fluid, relation between wave length and wave period, energy transport, etc.

\subsection{Regular wave}

The concept of regular wave is mainly adopted from the book written by J.M.J. Journée \& W.W. Massie ${ }^{[11]}$. The figure 1 shows a harmonic wave as seen from two different perspectives: Figure 1-a shows at a fixed time, the wave would be like a snapshot photo from the side of a transparent wave; the wave profile is shown as a function of distance $x$ along the propagation direction. Figure. 1-b is a time record of the water when the observed location is fixed: 

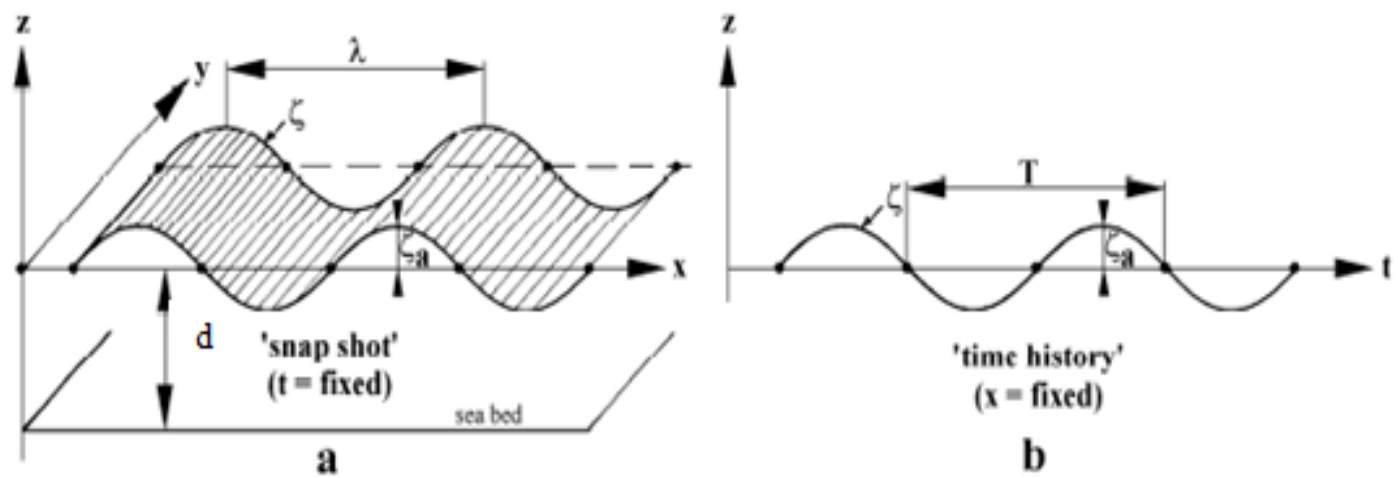

Figure 1. Harmonic wave definitions

Notice that In 1-a figure, $x$-axis lies at still water level, which is the average water level. z-axis directed upward, the water depth, $d$ is the vertical distance between still water level and seabed $(z=-d)$. The highest point of the wave is called crest while the lowest point is the trough. The wave length $\lambda$ is the horizontal distance between any two successive crests.

In Figure. 1-b, the distance between two successive crests in time domain is the wave period, T. The wave height is the distance between the crest and trough, denoted as $\mathrm{H}$.

Usually, if the wave is described by a sinusoidal function, wave amplitude $\zeta_{a}$ is necessary which is the half of the wave height. If the wave moves in the positive $x$ direction, the water surface elevation can be expressed as a function of both $x$ and $t$ as follows:

$$
\zeta=\zeta_{a} \cos (k x-\omega t)
$$

where

$\omega$ is angular frequency, relatede to wave period $T, \omega=\frac{2 \pi}{T}$;

$$
\text { kiswavenumber, related to wave length } \lambda, k=2 \pi / \lambda
$$

Eq. (1.1) could be considered a general description of almost any type of wave, depending only on the interpretation of $\zeta$.For further wave analysis, a liner approach is usually applied for simplification. It is important to recognise its limitation, especially when nonlinear effects become significant. However, this linear approach could work quite well for many practical problems. This linear wave theory is discussed in following section.

\subsection{Linear wave theory}

Linear wave theory, or Airy wave theory, is the core theory of ocean surface waves used in coastal engineering and naval architecture, which provides a linearized description of the propagation of a homogeneous fluid layer. This theory was first published in correct form by George Biddel Airy in $19^{\text {th }}$ century, it is established based on following assumptions ${ }^{[12]}$ :

\section{1) The sea water is incompressible}

2) The sea water is inviscid. Meaning friction effect is ignored.

3 ) There is no surface tension.

4) The sea water is irrotational flow.

5) The wavelength is much larger than wave amplitude. In other word, the wave amplitude is small.

6) The seabed is fixed, horizontal and impermeable.

Above assumptions are important in the linear wave theory, as they are the precondition for many derivations. The restriction that small wave amplitude is important. It states that the wave height must be very small compared to the wavelength. In other words, the wave must have a small slope. Only then the velocity of water particle equals to the velocity of wave surface thus Eq. (1.1) shall be a proper expression of a physical wave. The linear waves theory cannot be applied to explain things like breaking waves or secondorder wave strokes and the energy transfer between one wave to another. Nevertheless, as nonlinear wave theory is much more complex, this report is largely limited to linear waves. These equations assume that the wave is neither being forced nor 
dissipated.

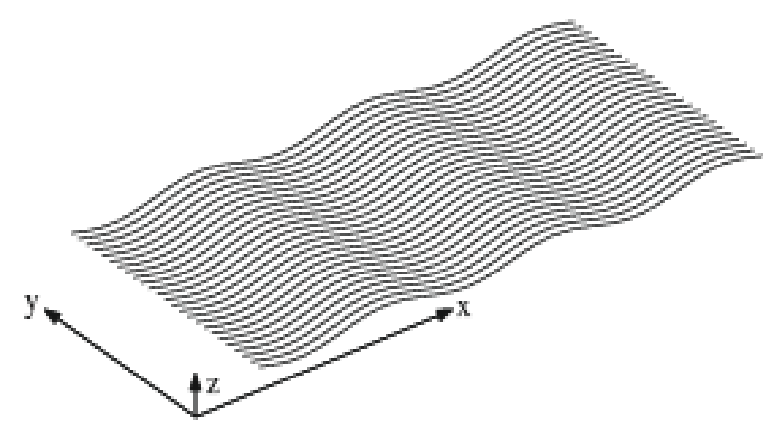

Figure 2. Space illustration of 2-D waves ${ }^{[13]}$

Consider a two-dimensional scenario of ocean surface wave. The wave propagates horizontally along the $x$-direction, the wave crests are parallel to the $y$-axis and

$z$-axis represents vertical direction. Let $u$ and $w$ be the horizontal ( $x$ direction) and vertical (z-direction) particle velocity respectively. Based on the first assumption -the fluid is incompressible, which allows the following equation to be derived: $\frac{\partial u}{\partial x}+\frac{\partial w}{\partial z}=0$

of the continuity equation [11,13]. Meanwhile, based on fourth assumption for irrotational flow, the references have provided another relation between $u$ and $w$ :

$\frac{\partial v}{\partial x}-\frac{\partial u}{\partial z}=0$

as a mathematical expression, whose derivative with respect to a chosen direction is the velocity

$u=\frac{\partial \Phi}{\partial x} ; w=\frac{\partial \Phi}{\partial z}$

this yields Laplace equation for an incompressible fluid:

$$
\frac{\partial^{2} \Phi}{\partial x^{2}}+\frac{\partial^{2} \Phi}{\partial z^{2}}=0
$$

To solve Eq.(1.4), a set of elevation and $z$ the vertical coordinate of sea surface measured form the still water level upwards. The kinematic condition at $z=0$ states that the vertical component at that direction:

velocity of water particle is equal to the vertical velocity of water surface:

$$
\frac{\partial \Phi}{\partial z}=\frac{\partial \zeta}{\partial t}
$$


This condition holds only if small wave amplitudes assumption is made ${ }^{[13]}$.

Bernoulli equation for irrotational

$$
-\frac{\partial \Phi}{\partial t}+\frac{1}{2}\left(u^{2}+w^{2}\right)+\frac{p}{\rho}+g z=0
$$

The value of $z$ apply the freesurface condition at sea surface where $\quad z=0$, surface displacement $Z$ is actually the

$$
-\frac{\partial \Phi}{\partial t}+g \zeta=0, \quad \text { at } z=0
$$

water elevation $\zeta$; water pressure equals to atmospheric pressure, meaning that $p=0$. Meanwhile, small wave amplitude assumption
Equation for irrotational flow in their paper, written as: allows the term of square of particle velocities to be neglected. Then Equ. (1.6) becomes:

From Eqs. (1.6) and (1.8), the linearized free-surface condition is

$$
\frac{\partial^{2} \Phi}{\partial t^{2}}+g \frac{\partial \Phi}{\partial z}=0
$$

Bottom boundary condition $(z=$ $-d)$ :The seabed is impermeable which means no flow can get through thus

$$
w=\frac{\partial \Phi}{\partial z}=0
$$

potential by solving Laplace equation in his book. The

V. Sundar ${ }^{[14]}$ has detailed the derivation for the velocity

$$
\Phi(x, z, t)=\frac{H}{2} \frac{g \cosh k(d+z)}{\omega \cosh k d} \sin (k x-\omega t)
$$

The wave potential expressed by Eq. (1.11) is very important, a lot of functions and quantities are dependent on this expression. For example, the wave elevation described at the very beginning of

expression is shown below:

$$
\zeta=\zeta_{a} \cos (k x-\omega t)
$$

This is why the regular waves have such expression.

Another important usage of wave potential is to establish the relationship between angular frequency $\omega$ and wave number $k$. Substitute the expression Eq. (1.11) for the wave potential into Eq. (1.9)
Part1, is a solution to Eq. (1.8) once wave potential is given:

$$
\omega^{2}=k \cdot g \cdot \tanh (k d)
$$


In many simulations, the angular frequency $\omega$ or wave period $T$ are given quantities while wave

Now the solution for $k$ is easy to find. In this report, dispersion equation is very handy to compute the value of $k$ in the floating body transfer function, this will be discussed in Part 2.

\subsection{Irregular Waves}

number $k$ or wavelength $\lambda$ must be determined. Under deep water condition, where water depth approaches to very large number

$\omega^{2}=k \cdot g$

Now, the basic knowledge of regular waves have be discussed in previous section. However, the observed sea surface seems never in a regular shape, they are unpredictable and irregular. Even so, they can be seen as a superposition of many simple, regular $\mathrm{h}$ wave components, each (we have $\tanh (k d)=1$ ), Eq. (1.13) becomes a simple form:

individual wave has its own frequency, amplitude, wavelength, period and propagation direction. This so-called superposition principle, which is first introduced in hydrodynamics by Denis and Pierson in 1953 and then adopted by J.M.J. Journée \& W.W. Massie ${ }^{[11]}$, is illustrated in Figure 3:

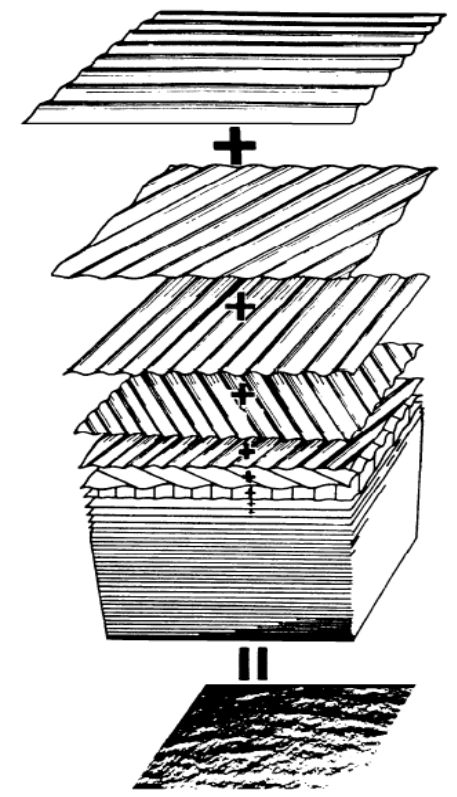

Figure 3. A sum of many simple sine waves makes an irregular sea ${ }^{[11]}$

This concept is widely used in offshore hydrodynamics field, as it allows the prediction of complex irregular behaviour becomes much simpler in terms of regular waves theory. Figure below explains how a time record of irregular wave is made up by 4 different sinusoidal waves: 


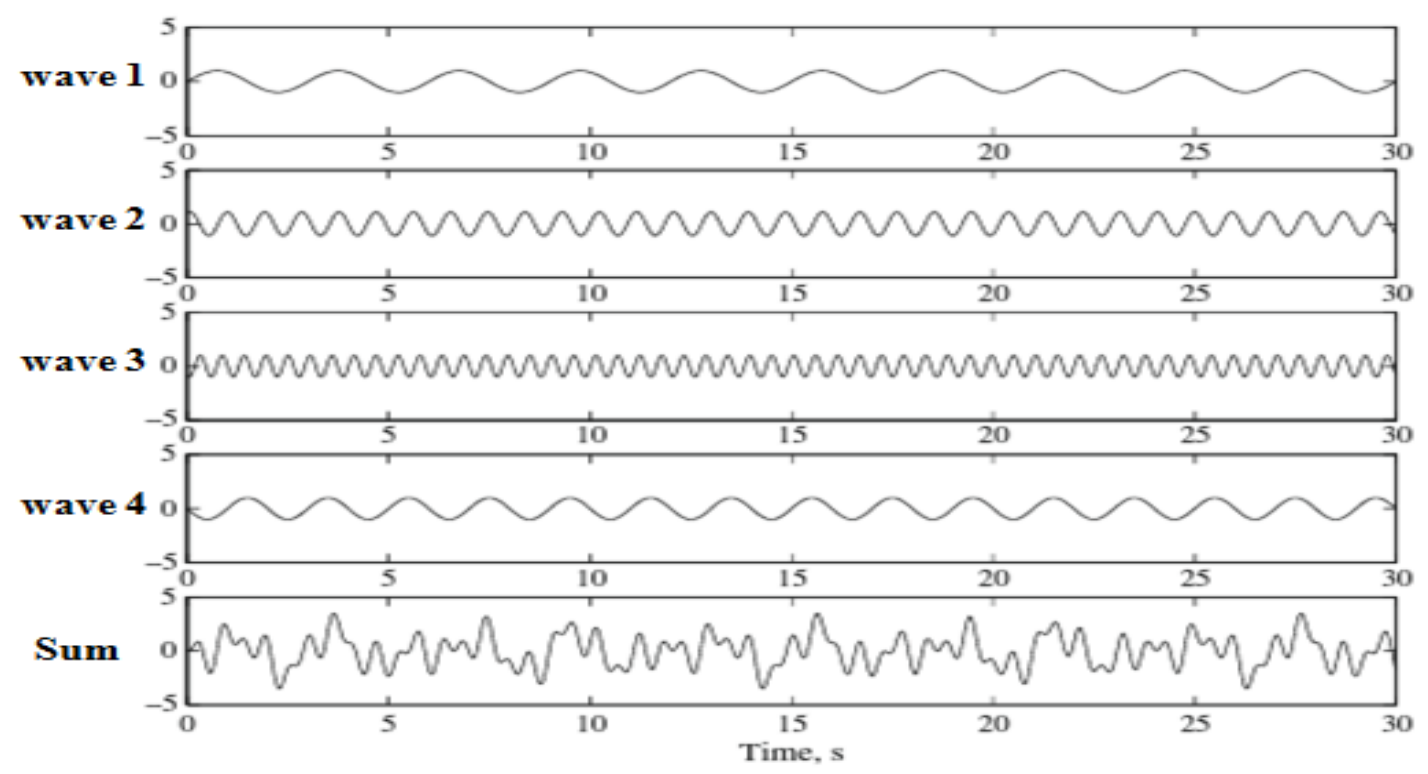

Figure 4. The superposition for four waves

Thus, linearized wave theory allows the wave elevation of a irregular sea along the $\mathrm{x}$-axis to be treated as a superposition of a series of regular waves, which the sea surface can be represented as:

$$
\zeta=\sum_{i=1}^{\mathrm{N}} \zeta_{a, i} \cos \left(k_{i} x-\omega_{i} t+\varepsilon_{i}\right)
$$

where for each component $\mathrm{i}$ :

$\zeta_{a, i}$ is the component wave amplitude;

$\omega_{i}$ is the component angular frequency;

$k_{i}$ is the component wave number;

$\varepsilon_{i}$ is the random phase angle.

However, it is not wise to express the sea state in terms of a series of regular waves as it might need numerous wave functions. Instead, smart engineers use Fourier series analysis to convert wave data from time domain to frequency domain, then they find a new quantity called wave spectrum to represent the highly confused pattern of waves.

\subsubsection{Wave spectrum energy density}

To obtain the wave spectrum, the fundamental concept is to decompose the measured irregular wave record to a finite number of single regular wave, then change the vertical axis from wave amplitude into an energy associated term $S_{\xi}$, following graph shows the basic concept of transformation: 


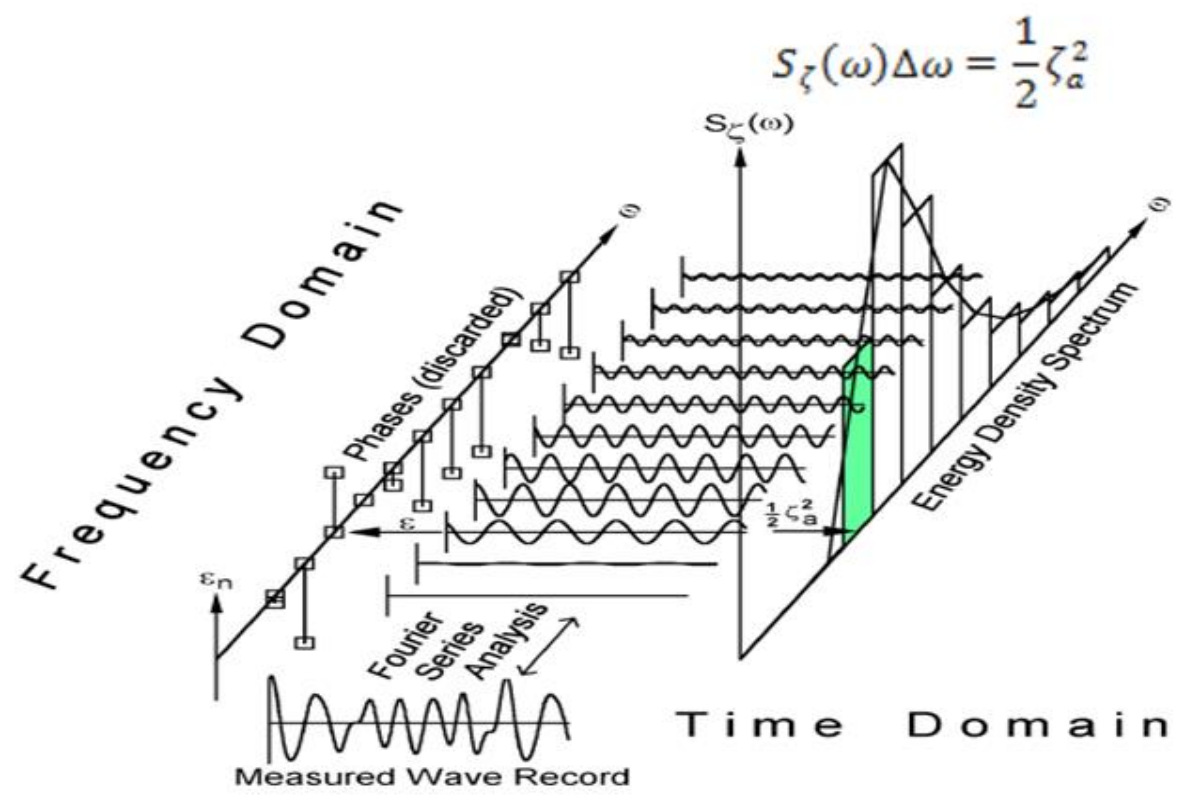

Figure 5. Wave record analysis

It can be seen in the figure above, the wave spectrum is a quantity which is related to the power of wave amplitude and by definition:

$$
S_{\zeta}(\omega) \Delta \omega=\frac{1}{2} \zeta_{a}^{2}
$$

Various formulations of wave spectrum have been developed from field measurements. The two most widely used forms are
Pierson-Moskowitz (PM) spectrum and JONSWAP spectrum. JONSWAP wave spectrum are chosen to represent the sea state in this report, it has a formulation of:

$$
S_{\xi}(\omega)=B\left(\frac{H_{S}}{4}\right)^{2} \frac{\omega_{p}^{4}}{\omega^{5}} \exp \left[-1.25\left(\frac{\omega}{\omega_{p}}\right)^{-4}\right] \gamma^{\Phi}
$$

Where

$H_{S}$ is the significant wave height; $\omega_{p}$ is the peak frequency

$$
\begin{aligned}
& \Phi=\exp \left[-\frac{1}{2 \beta^{2}}\left(\frac{\omega}{\omega_{p}}-1\right)^{2}\right] \\
& B=3.29 \\
& \gamma=3.3 \\
& \beta=\left\{\begin{array}{l}
0.07, \text { when } \omega \leq \omega_{p} \\
0.09, \text { when } \omega>\omega_{p}
\end{array}\right.
\end{aligned}
$$

The above formulation seems terrifying, actually, all the variables have been quantified by field measurements and act like a constant in the formulation. For a given set of wave data, the term significant wave height $H_{S}$ and peak angular frequency $\omega_{p}$ are known quantities. Now, the only variable in the formulation is the frequency $\omega$, therefore JONSWAP spectrum can seen as a frequency dependent function.

\subsubsection{Simple wave statistics}

JONSWAP spectrum has brought two new terms - significant wave height and peak angular frequency into discussion.

In Karsten's work, he gives the clear definitions to these two terms ${ }^{[15]}$ :

- The significant wave height, $H_{S}$, 
is the mean of the highest third of the waves in a time-series of waves representing a certain sea state.

- The peak wave frequency, $\omega_{p}$, is the wave period with the highest energy, which means that the wave with that frequency has the highest wave amplitude.

These quantities are included in wave statistics field, which is not the major concern in the report. One can easily find literatures or website which has elaborate introduction to wave statistics.

\section{Response characteristic}

The basic wave theory has been discussed in Part 1, which defines the input for floating body motion analysis. As shown in Figure. 6, the floating body performs as the transfer function which represent the body characteristics due to wave forces. In this case, the transfer function is so-called Response Amplitude Operator (RAO), which is usually obtained from mathematical modeling or proposed ship designs tested in a model basin, or from specialized simulation software.
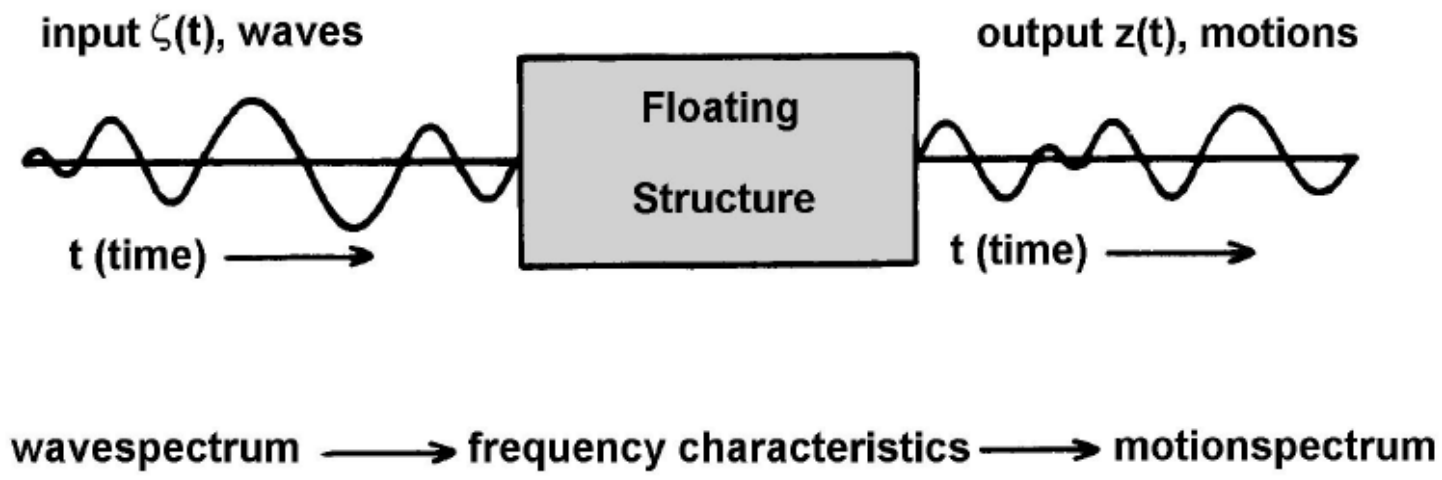

Figure 6. Relation between motions and waves ${ }^{[11]}$

\subsection{Motion convention}

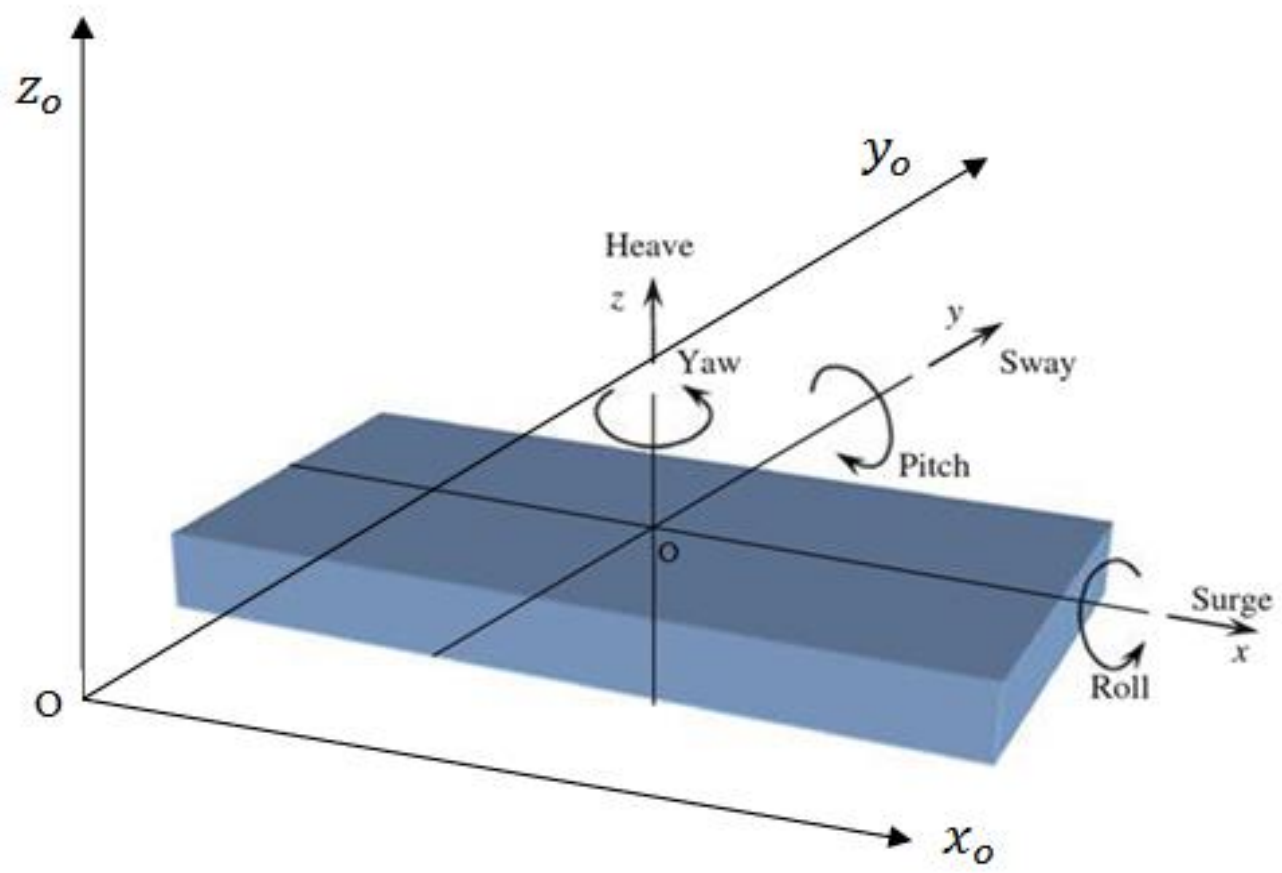

Figure 7. Coordinates convention and motion of floating structures ${ }^{[11]}$

In general, two orthogonal coordinate systems need to be defined before analyzing the 
motion of floating structure as shown in Figure 7. :

1) The earth-fixed coordinate system O $\left(x_{o} ; y_{o} ; z_{o}\right)$ The still water surface aligns with $x_{o}-y_{o}$ plane; the positive $x_{o}$-direction indicates the direction of wave propagation; the positive $z_{O}$-axis is directed upwards.

2) The body-fixed coordinate system $\circ(x ; y ; z)$.The origin of this system is the centre of gravity of the floating structure. Considering a ship moving in the ocean,

$x$-Axis indicates the longitudinal forward direction, $y$-deriction representsthe lateral side direction and $z$ is for the up-anddown motion.

(Note that: If the ship is only moving vertically in still water, the $x-y$ plane is parallel to the still water surface.

Figure also shows 6 motions of freedoms of a ship, the notations of these motions as well as the expression are given below:

Three translation motions are:

1) Surge: along the $\mathrm{x}$-axis, $x=$ $x_{a} \cos \left(\omega_{e} t+\epsilon_{x}\right)$

2) Sway: along the $y$-axis, $y=$ $y_{a} \cos \left(\omega_{e} t+\epsilon_{y}\right)$

3) Heave: along the z-axis, $z=$ $z_{a} \cos \left(\omega_{e} t+\epsilon_{z}\right)$

Three rotational motions are:

1) Roll: with respect to $x$-axis, $\emptyset=$ $\emptyset_{a} \cos \left(\omega_{e} t+\epsilon_{\varnothing}\right)$

2) Pitch: with respect to $y$-axis, $\theta=$ $\theta_{a} \cos \left(\omega_{e} t+\epsilon_{\theta}\right)$

3) Yaw: with respect to z-axis, $\gamma=$ $\gamma_{a} \cos \left(\omega_{e} t+\epsilon_{x}\right)$

Where, the term with subscript a represents the amplitude of each motion;

$\omega_{e}$ is the frequency that the floating body encounters the waves;

$\epsilon_{x}$ is the phase angle;

These 6 ship motions introduced above are body motions, specifically, is the relative motion of the body-fixed origin with respect to the earth-fixed system. However, the absolute motion are what engineers always interested in, which are the motions of the ship in earth-fixed coordinate systemo $\left(x_{o} ; y_{o} ; z_{o}\right)$.

Brian and López ${ }^{[13]}$ stated that it is easily understood that the absolute motion of arbitrary point on the structure is the resultant all 6 motions. Once the two coordinates and the motions are defined, the absolute motion can be seen as a superposition of relevant motions. However, this linearized superposition only holds when the value of rotational motion $\varnothing, \theta$ and $\gamma$ are small ( $\cos \emptyset=1$ and $\sin \varnothing=0$ ). Referred to Ship Hydrostatics and Stability, the vertical motion of the point is written as:

$$
z_{\text {absolute }}=z-x \theta+y \emptyset
$$

For simplicity, this report does not cover the coupled motion analysis, thus the last two terms are omitted from the equation. However, for more accurate modelling, which most papers did, the coupled motion analysis is concluded.

\subsection{Force Analysis}

In offshore engineering field, wave-induced force on floating structures is always an intriguing subject. One of the most accepted approach was proposed by Morison ${ }^{[16]}$, who wrote down the famous Morison's equation to characterize the wave-induced force as the sum of drag force and inertia force. This equation was modified and adopted by many later scholars, it has a original form of:

$$
F=\frac{1}{2} C_{D} \rho D u|u|+C_{M} \rho A \frac{d u}{d t}
$$

Where: $\quad u$ is the horizontal velocity of water particle;

$D$ is cylinder diameter; $\rho$ is the flow density;

$C_{D}$ is called drag coefficient; $C_{M}$ is called inertia coefficient.

From Eq. (2.1), it can be seen that the direction of the force is aligned with horizontal particle velocity, which indicates that the force has no contribution to heave motion if uncoupled motion analysis is taken. Thus Morison's equation is not necessarily involved in force analysis in this section. For more information regarding to Morison's Equation, one can find useful to read

The most widely used model to describe the vertical motion of floating object is the so-called Mass-Spring-Damper System 
(MSDS), which is considered to be linear. Thus, the analysis of the resulting motion in waves can be seen as a superposition of the motion of the object in still water and the forces on the restrained body in waves, a cylinder is used here to demonstrate the analysis as shown in Figure 2.3:
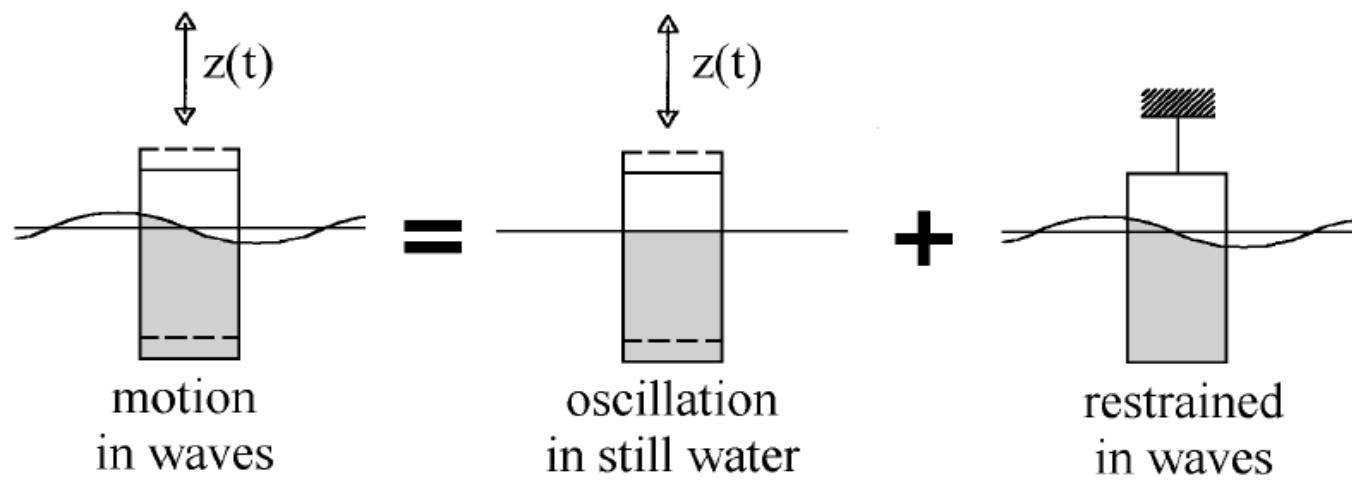

Figure 8. Superposition of hydromechanical and wave loads

Consequently, the force analysis can be broken into two circumstances:

1) Analyzing the forces caused by the oscillation of the rigid in still water, this is so-called hydromechanical forces, denoted by $F_{h}$.

2) Analyzing the force produced by incoming waves on the restrained body.

In most cases, moment equation is necessary. However, knowledge involved in this paper is simple, only the vertical motion is analyzed, thus the coupled motion analysis, meaning that the governing equation of motion in terms of moment is neglected. The governing equation of motion is given by Newton's Law,

$$
m \ddot{z}=F_{h}+F_{w}
$$

\subsubsection{Hydromechanical force}

The hydromechanical force exerted by the body motion in the water is analyzed from a free

decay test, as shown in Figure. 9: The cylinder is released at still water with an initial displacement, it will oscillates freely until energy dissipates and becomes stationary. During the process, the cylinder is only subjected to the hydromechanical force, the governing equation is shown below:

$$
m \ddot{z}=F_{h}
$$

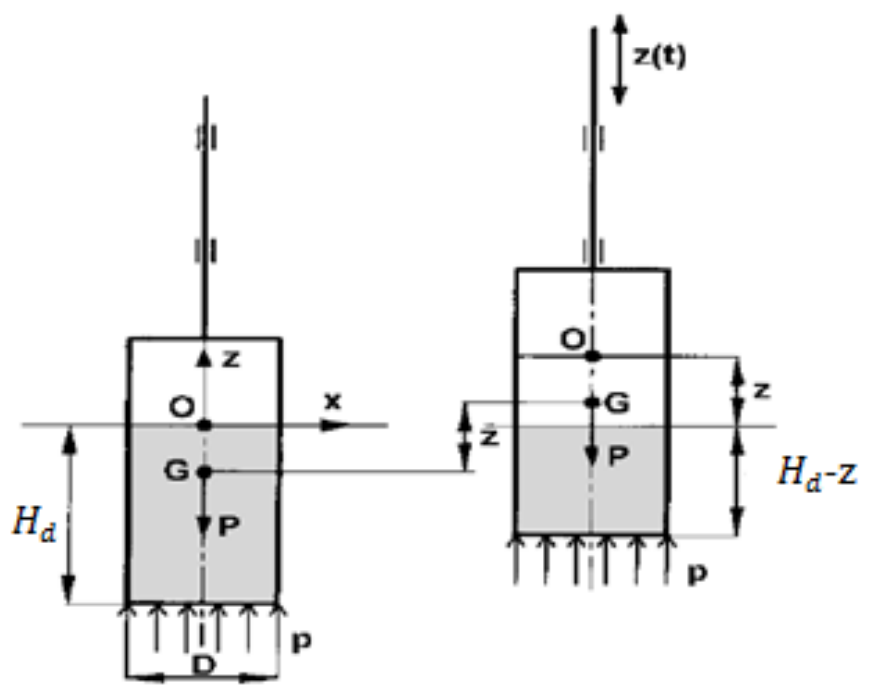


Figure 9. Cylinder oscillating in still water

The equation of motion is expressed as mass times acceleration on the left hand side equals to sum of force on the right hand side. This free decay test is similar to a spring-mass system, which must consider damping effect in force analysis. Also, a term associated with acceleration

$$
\begin{aligned}
m \ddot{z} & =-m g+p A-b \dot{z}-a \ddot{z} \\
& =-m g+\rho g\left(H_{d}-z\right) A-b \dot{z}-a \ddot{z} \\
& =-c z-b \dot{z}-a \ddot{z}
\end{aligned}
$$

Where: $\quad \mathrm{z}$ is the vertical displacement of the cylinder $(m)$;

$\rho$ is the flow density $\left(\mathrm{kg} / \mathrm{m}^{3}\right)$;

$\mathrm{A}$ is the cross-section area $\left(\mathrm{m}^{2}\right)$;

$\mathrm{H}_{\mathrm{d}}$ is the draft of cylinder at rest (m);

$\mathrm{m}$ is the mass of cylinder $(\mathrm{kg}), m=\rho A H_{d}$;

$m g$ is gravity downwards $(\mathrm{N})$;

$a$ is called added mass, or hydrodynamic mass ( $\mathrm{kg})$;

bis called hydrodynamic damping coefficient $(\mathrm{kg} / \mathrm{s})$;

$\mathrm{c}$ is restoring spring coefficient $\left(\mathrm{kg} / \mathrm{s}^{2}\right), c=\rho g A$;

In the free decay test, the hydromechanical $\mathrm{F}_{\mathrm{h}}$ is the only external force, now from Eq. (2.4) and Eq.(2.5), it is easy to write down the expression of

$$
F_{h}=-c z-b \dot{z}-a \ddot{z}
$$

The term $\mathrm{c}$ is always a constant value for a given object. However, added mass and damping coefficient are two terms which have been investigated for years.

\subsubsection{Added mass a \& damping coefficient $b$}

Added mass and damping are key factors in such motion response analysis. From early 1980s, a set of theoretical added masses and wave damping data for a floating circular cylinder in finite-depth water is presented. In Newman's book, he provided added mass for submerged 2-D bodies, however, the paper only considered the influence of shape, which is proved incorrect [17]. Ronald W Yeung made same achievement as he thought the added mass and damping was fixed if geometries were given ${ }^{[18]}$. As time goes by, the research continued. Kristiansen and Egeland ${ }^{[19]}$ once called hydrodynamic mass comes to the topic. The total force can be written as: hydromechanical force :

stated in their design that the added mass and damping are frequency- dependent variables. Filippos Kalofotias [20] has computed these coefficients using the BEM code $\mathrm{NEMOH}$, some of his models were adopted in this report.

\subsubsection{Froude-krylov}

In the second scenario, the cylinder is restrained, so that one now need to measure the vertical 
wave load on this fixed cylinder.

This is shown in Figure. 10:

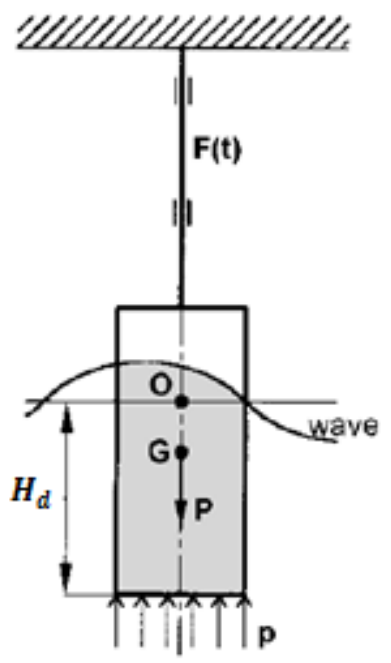

Figure 10. Cylinder restrained in waves

It can be seen that, the force acting on the cylinder is the water pressure generated by the wave towards upright and restrained force which hold body into stationary state downwards. To figure out the restrained force, the water pressure is necessarily to be defined first, we need recall water

Wave potential: $\quad \Phi=\frac{\zeta_{a} \mathrm{~g}}{\omega} e^{k z} \sin (k x-\omega t)$

Wave elevation: $\quad \zeta=\zeta_{a} \cos (k x-\omega t)$

So that the pressure, $\mathrm{p}$, on the $-H_{d}$ )follows from the linearized bottom of the cylinder $(\zeta=$ Bernoulli equation:

$$
p=\rho g \zeta_{a} e^{-k H_{d}} \cos (\omega t)+\rho g T
$$

wave length, so that the pressure field is completely undisturbed by the presence of the cylinder as Assuming that the diameter of the cylinder is small relative to the well as essentially uniform potential and wave elevation then substitute into Bernoulli equation, that is:

$$
p=\rho g \zeta_{a} e^{-k H_{d}} \cos (\omega t)+\rho g T
$$

The vertical force equals to the pressure times cross-section area thus equals to:

$$
F=\left[\rho g \zeta_{a} e^{-k H_{d}} \cos (\omega t)+\rho g T\right] \cdot \frac{\pi}{4} D^{2}
$$

where $D$ is the cylinder diameter; $\quad H_{d}$ is the draft. 
the regular harmonic wave force, which will be considered here. This wave force is called Froude-Krylov force can also be expressed in a similar way with the hydromechanical loads, which is a spring coefficient term $\mathrm{c}$ times a effective wave elevation $\zeta^{*}$ :

$$
\text { where } c=\rho g \frac{\pi}{4} D^{2} ; \zeta^{*}=\zeta_{a} e^{-k T} \cos (\omega t)
$$

Modify the Froude-Krylov force by adding up added mass and proportional to the vertical acceleration and vertical velocity damping term, which are of the water particles, the total

wave force is rewritten as:

$$
F_{w}=a \ddot{\zeta}^{*}+b \dot{\zeta}^{*}+c \zeta^{*}
$$

Where:

$$
\begin{aligned}
& \zeta^{*}=\zeta_{a} e^{-k T} \cos (\omega t) \\
& \dot{\zeta}^{*}=-\zeta_{a} e^{-k T} \omega \sin (\omega t) \\
& \ddot{\zeta}^{*}=-\zeta_{a} e^{-k T} \omega^{2} \cos (\omega t) ;
\end{aligned}
$$

Substitute Eq.(2.14) in Eq.(2.13) yields:

$$
F_{w}=\left(c-a \omega^{2}\right) \zeta_{a} e^{-k T} \cos (\omega t)-(b) \zeta_{a} e^{-k T} \omega \sin (\omega t)
$$

\section{Response in waves -- RAO}

Recall the equation of motion in part 2, this time the total force is

the sum of hydormechanical force and Froude-Krylov force:

$$
m \ddot{z}=F_{h}+F_{w}
$$

As discussed before, $F_{h}$ is expressed in Eq.(2.6) and $F_{w}$ is

expressed in Eq.(2.13). Substitute expression of $F_{h}$ and $F_{w}$ into
Eq.(2.16), yields

Where

$$
m \ddot{z}=a\left(\ddot{\zeta}^{*}-\ddot{z}\right)+b\left(\dot{\zeta}^{*}-\dot{z}\right)+c\left(\zeta^{*}-z\right)
$$

$$
\begin{aligned}
& z=z_{a} e^{-k H_{d}} \cos \left(\omega t+\varepsilon_{z}\right) \\
& \dot{z}=-z_{a} e^{-k H_{d}} \omega \sin \left(\omega t+\varepsilon_{z}\right) \\
& \ddot{z}=-z_{a} e^{-k H_{d}} \omega^{2} \cos \left(\omega t+\varepsilon_{z}\right) \\
& \zeta^{*}=\zeta_{a} e^{-k H_{d}} \cos (\omega t) \\
& \dot{\zeta}^{*}=-\zeta_{a} e^{-k H_{d}} \omega \sin (\omega t) \\
& \text { Distributed under creative commons license 4.0 }
\end{aligned}
$$




$$
\ddot{\zeta}^{*}=-\zeta_{a} e^{-k H_{d}} \omega^{2} \cos (\omega t)
$$

Substitute Eq.(2.18) into Eq.(2.17), in :

$$
\frac{Z_{a}}{\zeta_{a}}=e^{-k \cdot H_{d}} \sqrt{\frac{\left(c-a \omega^{2}\right)^{2}+(b \omega)^{2}}{\left\{c-(m+a) \omega^{2}\right\}^{2}+(b \omega)^{2}}}
$$

Where

$$
\begin{aligned}
& Z_{a} \text { isheaveamplitude; } \\
& \zeta_{a} \text { iswaveamplitude } \\
& H_{d} \text { is draft of body at rest } \\
& \text { kiswavenumber, which could be calculated through dispersion } \\
& \text { relation with } \omega . \\
& \text { a is added mass; } b \text { is damping coefficient } \\
& c \text { is restoring spring coefficient; } \text { wisfrequency }
\end{aligned}
$$

The expression $\frac{Z_{a}}{\zeta_{a}}$ describe how heave amplitude $Z_{a}$ is proportional to the wave amplitude $\zeta_{a}$. One may notice that the phase terms are discarded, this is because the phase is not dependent on the wave amplitude. This ratio or expression is so-called Response amplitude operator

Similarly, motion spectrum is related to the heave amplitude, it
Now we want the heave motion spectrum to be something related

$$
S_{\zeta}(\omega) \Delta \omega=\frac{1}{2} \zeta_{a}^{2}
$$

(RAO). It is the transfer used to determine the body response. The RAO of various motion could be obtained from coupled motion analysis ${ }^{[4]}$. In other word, one can determine RAO for all six motions of the floating body. In this report, the $\frac{z_{a}}{\zeta_{a}}$ is the RAO of heave motion. has the same form as wave spectrum while just replace the wave amplitude by heave

$$
S_{Z}(\omega) \Delta \omega=\frac{1}{2} z_{a}^{2}
$$

to the RAO, this is achieved by shifting the term $z_{a}^{2}$ into $\zeta_{a}^{2} \cdot\left|\frac{z_{a}^{2}}{\zeta_{a}^{2}}\right|$,

\section{Motion-Response-Motion spectrum}

Part 1 has introduced the concept of wave spectrum, recall the definition of wave spectrum, it is related to half the power of wave amplitude:

amplitude: while $\left|\frac{z_{a}^{2}}{\zeta_{a}^{2}}\right|$ is actually the square of RAO. This could help us to 


$$
\begin{aligned}
S_{Z}(\omega) \Delta \omega & =\frac{1}{2} \zeta_{a}^{2} \cdot\left|\frac{z_{a}^{2}}{\zeta_{a}^{2}}\right| \\
& =S_{\zeta}(\omega) \Delta \omega \cdot R A O^{2}
\end{aligned}
$$

Cancel out the term $\Delta \omega$ at both sides in Eq. (2.21) yields the relationship between wave spectrum, RAO and motion

$$
S_{Z}(\omega)=S_{\zeta}(\omega) \cdot R A O^{2}
$$

To conclude; with a given recorded wave data, once RAO of a concerned motion is determined, one can characterize the resulting motion by the motion spectrum.

\section{Results}

In part 2, RAO of heave motion is discussed. To predict the first spectrum:

order vessel motion caused by a particular wave spectrum, the motion response spectrum $S_{Z}(\omega)$ is written as:

- Peaking period: $12 \mathrm{~s}$

One simple model has been established to demonstrate how this particular approach works.

\subsection{Define input -- wave}

$$
S_{Z}(\omega)=S_{\zeta}(\omega) \cdot R A O^{2}
$$

\section{Spectrum}

Considering an irregular sea state which could be defined by the following parameters:

- Significant wave height: $8 \mathrm{~m}$
With given parameters, the corresponding wave spectrum could be represented on the basis of JONSWAP spectrum:

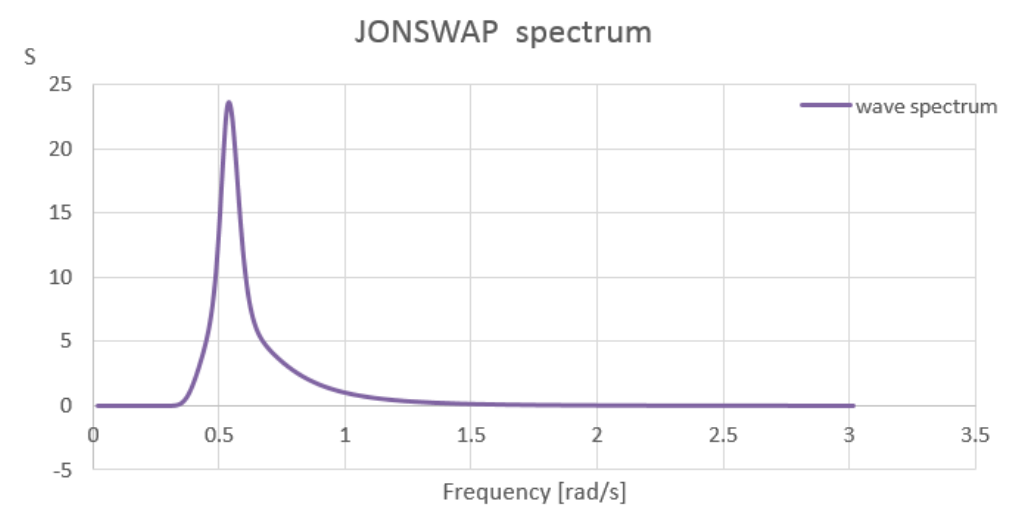

Figure 11. JONSWAP wave spectrum

\subsection{Define transfer function -- RAO}

Recall Eq. (2.19), which gives the response amplitude operator (RAO) for heave motion:

$$
\frac{Z_{a}}{\zeta_{a}}=e^{-k \cdot H_{d}} \sqrt{\frac{\left(c-a \omega^{2}\right)^{2}+(b \omega)^{2}}{\left\{c-(m+a) \omega^{2}\right\}^{2}+(b \omega)^{2}}}
$$


This equation indicates that the $R A O$ is a frequency dependent function. $H_{d}$ is the draft in still water, $\mathrm{k}$ is the wave number that can be solved by dispersion equation for each frequency. $c$ is the restoring spring term, that is a fixed value one the object is determined.

One tricky part lies in the values of added mass $a$ and damping coefficient $b$ in the function, because these are two frequency dependent quantities as well. Thus to figure out the value of $a$ and $b$ against frequency is the premise of the determination of RAO.

In Filippos Kalofotias's literature ${ }^{[20]}$, he used CFD simulation and boundary-element method (BEM) code called NEMOH to calculate the added mass and damping coefficients for three simple geometries. These three objects are a cylinder, a bullet and a cone while they have same diameter equals to $10 \mathrm{~m}$. Three geometries are introduced below:

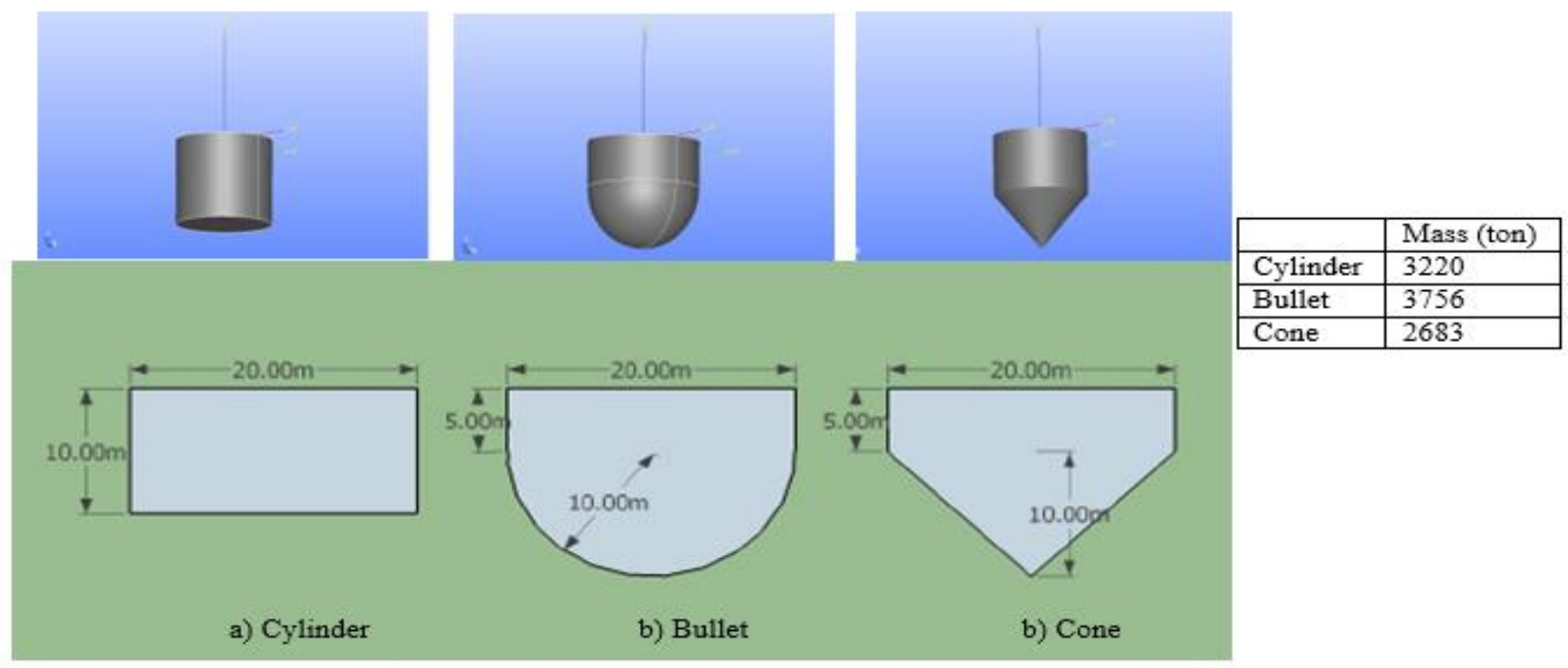

Figure 12. Basic parameter of cylinder, bullet and cone

Referring to Filippos, the plots of added mass $a(\omega)$ and damping coefficient $b(\omega)$ are

given below:

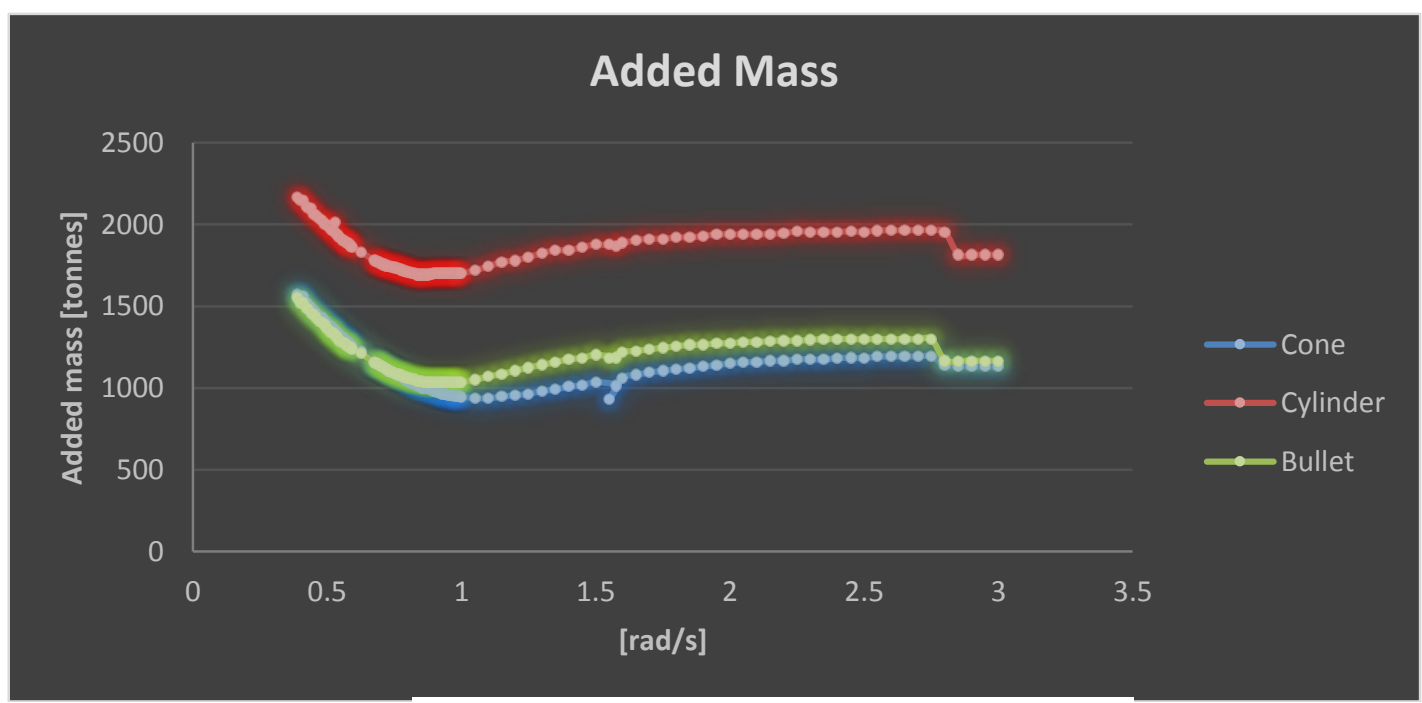

a. Added mass 


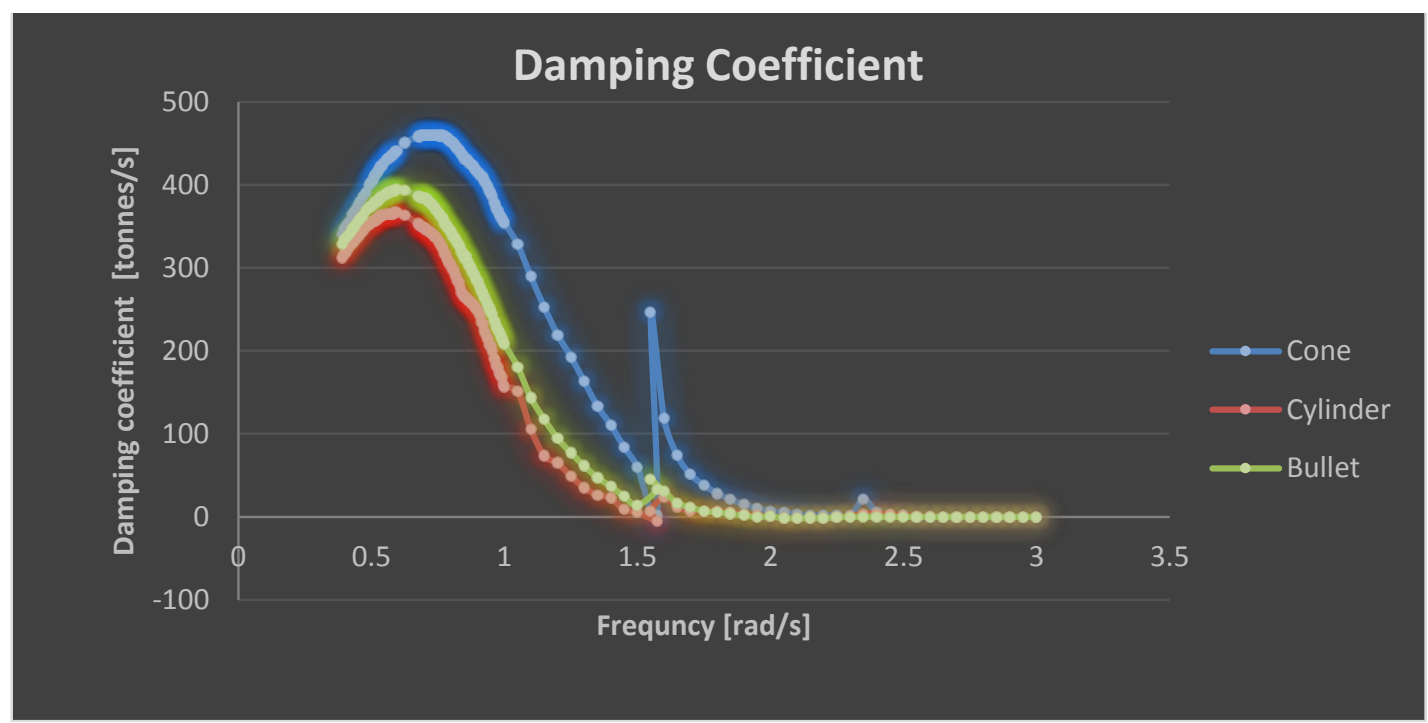

b. Damping coefficient

Figure 13. Added mass\& damping coefficients of the cylinder, bullet and cone

In added mass plot, the variation trend for all three objects are basically same. The cylinder has the largest added mass, the value decreases from 2200 [tonnes] and hit bottom when frequency reaches $1[\mathrm{rad} / \mathrm{s}]$, then it slightly increases and retain 2000 [tonnes] until $2.8[\mathrm{rad} / \mathrm{s}]$, at $2.8[\mathrm{rad} / \mathrm{s}]$ it has a clear drop down to 1800 [tonnes]. The added masses of bullet and cone are close to each other while bullet is slightly larger, they have a range between 1600 [tonnes] and 1300 [tonnes].

As for damping coefficient, the cone has the largest damping effects among three objects, the value rises from 300 [tonne/s] to 450 [tonne/s] as frequency increases from $0.3[\mathrm{rad} / \mathrm{s}]$ to 0.6 $[\mathrm{rad} / \mathrm{s}]$, then decreases to almost 0 when frequency reaches 1.5 $[\mathrm{rad} / \mathrm{s}]$, it is noticeable that there is a clear bounce back from 0 to 260 [tonnes/s] when frequency gets larger than $1.5[\mathrm{rad} / \mathrm{s}]$, then the damping coefficient drop again and gradually approach to 0 again. The cylinder and bullet seems less effected by damping, their peak damping coefficients are less than 400 [tonnes/s] at frequency of 0.6 $[\mathrm{rad} / \mathrm{s}]$, then decrease to 0 .

Substitute the known factors into the RAO function Eq. (3.2), which gives the plot of heave motion $R A O(\omega)$

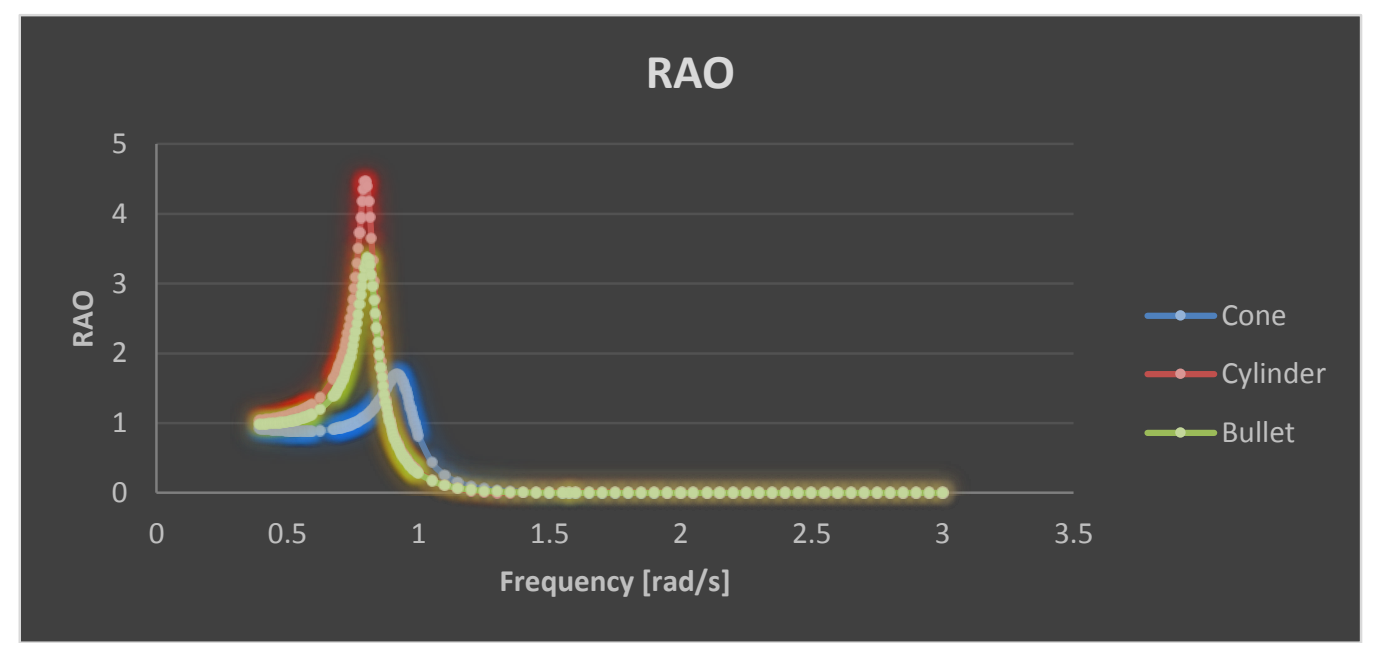

Figure 14. RAO of the cylinder, bullet and cone

From the graph, for cylinder and bullet, the RAO peaks at 4.5 with the frequency of $0.7[\mathrm{rad} / \mathrm{s}]$ while the cone peaks at 3.5 when frequency is $0.9[\mathrm{rad} / \mathrm{s}]$. The RAO of cone is much more smooth among three geometries, the peak value is only 1.5 when frequency reaches $1[\mathrm{rad} / \mathrm{s}]$. Now the transfer function spectrum is known, motion spectrum is easily obtained.

\subsection{Generate Output -- Motion Spectrum}

Recall Eq. (3.1 ), wave spectrum and motion spectrum is connected 
by the body transfer function RAO. Now the motion spectrum is easily determined with results obtained

from Step 1 and step 2, as shown in figure below:

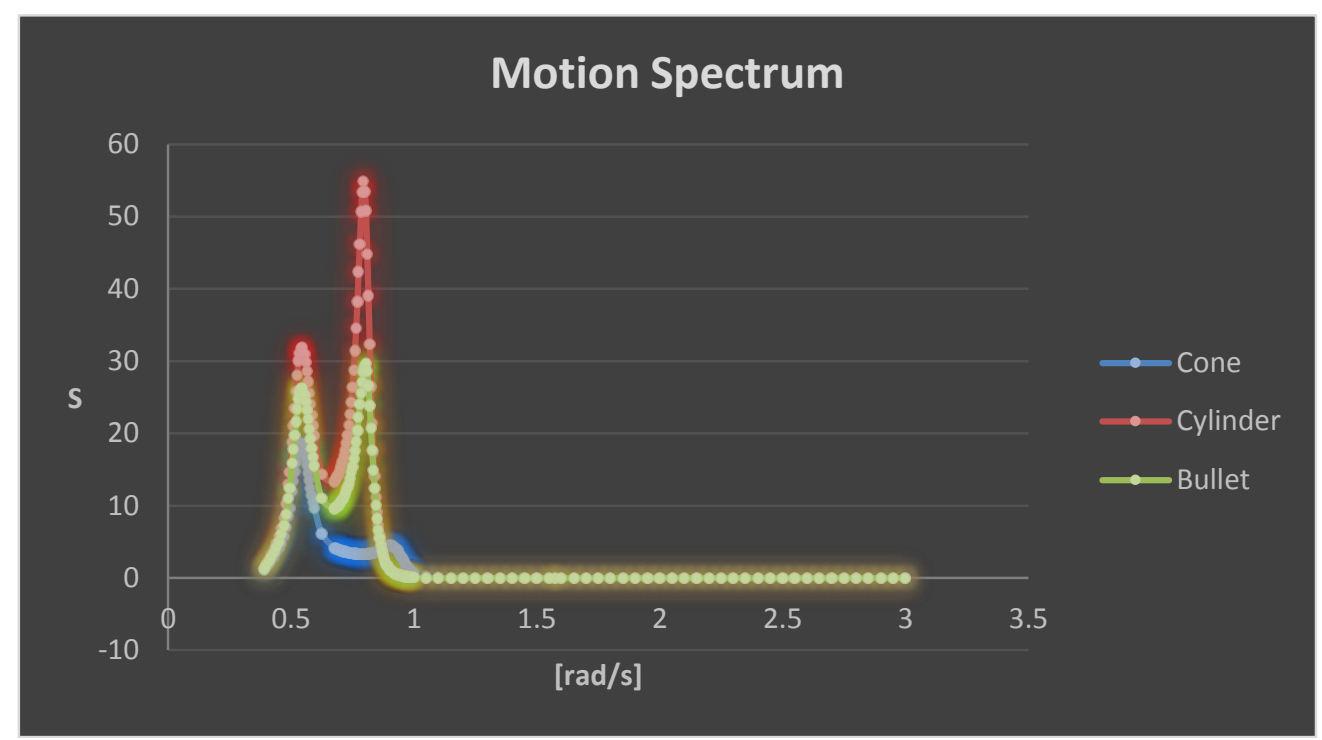

Figure 15. Motion spectrum of the cylinder, bullet and cone

The heave motion spectrums for three given objects are obtained. However, the main purpose of the report is to demonstrate how to use RAO to obtain the analyze response motion. As for how the motion spectrum is, what can be analyzed from the motion spectrum could be saved for future work.

\section{Discussion}

First of all, the input is obtained based on the linear wave theory, which assumes the wave behave linearly. Therefore, the irregular wave could be seen as a superposition of multiple harmonic regular waves. However, nonlinear waves are common nature such as stroke waves or waves generated in severe weather. In fact, as the problem shifted to near shore when the water becomes shallow, the waves starting behave more nonlinear. Therefore, the input in this RAO method has already make things ideal and might be lack of accuracy. Also, response amplitude operator has the same limitation as the linear wave theory because they both adopt the superposition principle, which could only be used for linearization. Another problem lies in the uncoupled motion analysis, the uncoupled motion is way too ideal, which is not close to real motion. A couple of literatures have covered the flaw in their analysis:

H.D. Zhang and C. Guedes Soaresstudies ${ }^{[21]}$ analyzed the behaviour of an LNG carrier and a chemical tanker encountering different long crested nonlinear water waves produced numerically by the nonlinear Schrödinger equation, which is capable of representing the modulational instability and of generating abnormal waves

Liu el at. ${ }^{[8]}$ have provided nonlinear force analysis for a Spar platform associated with 2 ndorder wave loads, also the heavepitch coupled motion is taken into account.

\section{Conclusion}

This paper provides a comprehensive review regarding to the prediction of heave motion in frequency domain analysis. The fundamental idea is to utilize a quantity which could define the irregular sea state as the input, then quantify the floating body characteristic with a transfer function, finally to obtain the output which is the response motion.
Initially, such basic knowledge regarding to regular wave and linear wave theory is introduced. The real sea state is more complicated and irregular. The concept of irregular wave is then addressed based on linearized superposition theory. In order to define the irregular sea state, engineers bring so-called wave spectrum or energy density spectrum which requires Fourier series analysis to convert the timedomain wave data into frequency domain, also one of the most widely used spectrum formJONSWAP spectrum is selected to use in this paper.

In many cases the floating structure mainly have a linear behaviour, based on the linearized condition, the overall motion can be seen as a superposition of the behaviours at each frequency. Thus, a frequency-dependent transfer function is desired. The transfer function is generally known as Response Amplitude Operator (RAO), which is usually obtained from software simulation or proposed ship designs tested in a model basin. Since the heave motion covered this report is quite simplified, the RAO is obtained through a wave-induced force analysis. During the force analysis, the associated terms added mass 
and damping coefficient are discussed.

In the end, based on the presented content, this report includes an example to show how RAO can be used to obtain the motion spectrum. The motion spectrum of three simple geometries are obtained with known hydrodynamic coefficients and assumed wave data. However, this approach has its limitations. First, the motion response in time domain is desired, thus one need to find a way to convert the motion spectrum back into time domain. Also, such linearization hypothesis and un-coupled motion analysis makes the problem way too ideal, which might lack of accuracy. This leads to future work which requires more supporting experiments to validate the method. Another future work could be find a proper way to convert the motion spectrum from frequency domain results into a motion time history.

\section{References:}

[1] Mostafa, N., Murai, M., Nishimura, R., Fujita, O. and Nihei, Y. (2012). Study of motion of spartype floating wind turbines in waves with effect of gyro moment at inclination. Journal of Naval Architecture and Marine Engineering, 9(1).

[2] Rho JB, Choi HS, 2004. Vertical motion characteristics of truss spars in waves. The Fourteenth International Offshore and Polar Engineering Conference, Toulon, France, 662-665.

[3] Tao LB, Lim KY, Thiagarajan K, 2004. Heave response of classic spar with variable geometry. Journal of Offshore Mechanics and Arctic Engineering, 126(1), 90-95.

[4] Hong YP, Lee DY, Choi YH, Hong SK, Kim SE, 2005. An experimental study on the extreme motion responses of a spar platform in the heave resonant waves. The Fifteenth International Offshore and Polar Engineering Conference, Seoul, Korea, 225-232.

[5] Neves MA, Sphaier SH, Mattoso $B M$, Rodríguez CA, Santos AL, Vileti VL, Torres FG, 2008. On the occurrence of Mathieu instabilities of vertical cylinders. The 27th InternationalConference on Offshore Mechanics and Arctic Engineering, Estoril, Portugal, 619627.

[6] Zhao Jingrui, Tang Lizhi, Tang Yougang, Wang Wenjie, 2009. Internal resonant responses of heave-pitch coupled motions of classic spar platform. Journal of Tianjin University, 42(3), 201-207.

[7] Shen Wenjun, Tang Yougang, 2011. Stochastic analysis of nonlinear coupled heave-pitch motion for the truss spar platform. Journal of Marine Science and Application, 10(4), 471-477.

[8] Liu, S., Tang, Y. and Li, W. (2016). Nonlinear random motion analysis of coupled heave-pitch motions of a spar platform considering 1st-order and 2ndorder wave loads. Journal of Marine Science and Application, 15(2), pp.166-174.

[9] Kurian VJ, Montasir OA, Narayanan SP, 2009. Numerical and model test results for truss spar platform. The Nineteenth International Offshore and Polar Engineering Conference,Osaka, Japan, 99-104.

[10] Li, B.B and Ou, J.P., 2009, Heave response analysis of truss Spar in frequency domain, The Ocean Engineering, 27(1):8-15.(in Chinese)

[11] J.M.J. Journée \&W.W. Massie, 2001. Offshore hrydrodynamics, Delft University of Technology.

[12] Craik, A. (2004). The Origins of Water Wave Theory. Annual Review of Fluid Mechanics, 36(1), pp.1-28.

[13] Biran, A. and López-Pulido, R. (2014). Ship hydrostatics and stability, second edition. 1st ed. Kidlington, Oxford, UK: Butterworth-Heinemann.

[14] Sundar, V. 2016. Ocean wave mechanics. John Wiley\& Sons Ltd.

[15] Mangor, Karsten (2007): Statistical

description of wave parameters. Available from: http://www.coastalwiki.org/wiki/S tatistical_description_of_wave_pa rameters [accessed on 28-05-2017] [16] Morison, J. R.; O'Brien, M. P.; Johnson, J. W.; Schaaf, S. A. (1950), "The force exerted by surface waves on piles", Petroleum Transactions, American Institute of Mining Engineers, 189: 149154, doi:10.2118/950149-G

[17] Pierson, W., Neumann, G. and James, R. (1984). Practical methods for observing and forecasting ocean waves by means of wave spectra and statistics. 1st ed. [Washington]: U.S. Naval Oceanographic Office.

[18] Yeung, R. W. \& Tan, W. T. (1980), "Hydrodynamic Interaction of Ships with Obstacles in Shallow Water", Journal of Ship Research, 24 (1): 50-59

[19] Kristiansen, E. and Egeland, O. (2003). Frequency-Dependent Added Mass in Models for Controller Design for Wave Motion Damping. IFAC Volumes, 36(21), pp.67-72.

[20] Filippos Kalofatias, 2016, Study for the Hull Shape of a Wave Energy converter-Point Absorber, University of Twente.

[21] Zhang, H. and Guedes Soares, C. (2016). Ship responses to abnormal waves simulated by the nonlinear Schrödinger equation. Ocean 119,143-153. 